\title{
L'IMAGINAIRE DANS LES ÉCRITS DES APPRENANTS : ENJEUX ET DÉMARCHES DIDACTIQUES EN CLASSE DE FRANÇAIS
}

\author{
Rachid El ALAOUI \\ Université Mohammed V, Maroc \\ Rachid.alaoui@um5s.net.ma \\ Article reçu le 3 octobre 2017 | révisé depuis le 8 octobre 2017 | accepté le 28 décembre 2017
}

\begin{abstract}
RÉSUMÉ. Cet article s'inscrit dans la lignée des travaux de recherche contemporains en didactique de l'écrit, dont ceux consacrés notamment à la question du sujet lecteurscripteur. L'objectif est de poser les jalons à la fois théoriques et méthodologiques d'un dispositif qui peut remédier aux difficultés motivationnelles des apprenants en classe de français langue étrangère, en considérant amplement l'apport de l'imaginaire dans leurs propres écrits. L'article s'accotera alors sur l'analyse d'un corpus d'écrit élaboré par des apprenants, suivi par ricochet d'une démarche de réécriture visant à proposer une interprétation des stratégies d'investissement par le sujet-apprenant. Les résultats ont montré que l'on a réussi alors à intégrer totalement le plan de l'imagination et de la créativité au sein des écrits rédigés par les apprenants, mais on a constaté aussi une appréciation de presque $40 \%$ pour ce qui est de l'aspect formel et structurel propre à la langue française.
\end{abstract}

Mots-clés : didactique de l'écrit, écriture d'invention, sujet lecteur-scripteur, réécriture, transposition didactique

\begin{abstract}
This article is a part of contemporary researches in didactics of writing, especially those who are based on the question of reader-writer subject. The study aims to overcome, theoretically and methodologically speaking, students' motivation difficulties in French class as a foreign language, by taking into didactic account the part of imagination in writing, beside its strictly linguistic or technical dimensions. This study investigates the corpus of students' writing productions, also it will examine the effects of a rewriting phase on investment strategies of subjects in their writings. The study shows that we have integrated the plan of imagination and creativity into the writings prepared by the learners, but also we have recorded an improvement of nearly $40 \%$ in terms of formal and structural aspect of the French language.
\end{abstract}

Keywords : didactics of writing, invention writing, reader-writer subject, rewriting, didactical transposition 


\section{INTRODUCTION}

La maîtrise de l'écrit en classe de français est d'une importance capitale du fait que la lecture et l'écriture constituent en soi, deux dimensions essentielles au cœur des pratiques d'enseignement et d'apprentissage. Le savoir-écrire, au même titre que le savoirlire présuppose, au-delà de la simple maîtrise de la langue française, une connaissance de soi, de l'autre, mais aussi du monde, et ce pour deux raisons capitales :

D'une part, les pratiques scripturales ne se réduisent pas uniquement à leur simple forme scolaire. D'autre part, l'écrit ne peut que dépasser le cadre des techniques rédactionnelles ordinaires, pour s'inscrire effectivement dans une véridique perspective constructiviste, il s'agit d'une expérience langagière ancrée dans des contextes institutionnels et disciplinaires particuliers, et qui tente d'élaborer et de suivre un sens bien précis et une appropriation complète d'un savoir bien déterminé.

L'intérêt colossal et primordial de l'apport de la didactique de l'écrit est de permettre à l'apprenant d'affirmer sa singularité dans un écologisme standardisé à grande échelle, et ce par les pratiques scripturales. En tant que sujet-apprenant défini par une culture, des représentations, des rapport(s) à soi, aux autres et même au monde de façon la plus globale.

C'est cette singularité même, qui définit la notion du sujet scripteur, puisqu' « on peut voir que la notion de sujet scripteur est souvent liée à la nécessité revendiquée de considérer la singularité du sujet, son investissement dans l'écriture, le sens qu'il donne à l'activité, sa « parole ». Elle est aussi articulée à la théorisation d'un sujet clivé et d'une langue non instrumentalisée. " (Delcamber, 2007, p.33).

À l'autre versant, on ne peut pas très bien-écrire en français sans la connaissance et la maitrise de ses dimensions linguisticodiscursives, mais l'écriture renvoie aussi vers une pratique sociale mêlant à la fois prescriptions institutionnelles, connaissances réelles, représentations de soi et du monde, valeurs et affects, contraintes linguistiques et opérations discursives, à des fins de construction du savoir (Reuter, 1996, p.58).
Ce faisant, on ne devrait nullement chérir une vision réductrice de l'apprenant vis-à-vis de la pratique scripturale, et aux différentes formes d'échec et d'insuccès propre à l'élève et qui est issu largement d'un manque de "savoir écrire » voire même de la résistance à apprendre à écrire en classe de français (Blaser et Chartrand, 2008, p.44). $C^{\prime}$ est ainsi que la dimension socio-affective de l'écriture pèse aussi lourdement pardevers l'aspect cognitif au sein même de l'activité scripturale.

L'élève détient alors des savoirs et des connaissances implicites sur lesquels il faudrait s'appuyer en classe de la langue française, et ce dans la mesure de maitriser ces propres déplacements significatifs dans les manières de penser l'apprentissage de l'écriture en classe de français, comme un lieu de tension réelle entre l'objets référés au récit et de l'autre, l'imaginaire des sujets.

On peut formuler la problématique de la manière suivante : est-ce qu'une consigne d'examen qui prône partiellement ou globalement la liberté de rédaction peut agir sur la place et le rôle de l'imaginaire dans des textes qui sont produits par les apprenants en cycle primaire marocain?

Le but ultime de notre article est de contribuer à la mise en place théorique et méthodologique d'un dispositif axé sur la prise en compte de la part de l'imaginaire dans les écrits des élèves en classe de français, et comme un variable clé et déterminant, permettant de développer amplement la motivation de l'apprenant et de faire évoluer par conséquent son implication au sein de la classe. On cherche alors à atteindre conséquemment deux objectifs primordiaux: D'emblée, on va tenter de repérer les difficultés des élèves en production de récits d'imagination et essayer de prouver en deuxième lieu, que la valorisation du rôle de l'erreur dans l'apprentissage de l'écrit influe sur les stratégies d'investissement au niveau des pratiques scripturales. Nous cherchons alors à montrer que la prise en compte de cette dimension constitutive de l'écriture scolaire constituerait l'une des voies possibles pour remédier aux difficultés motivationnelles des élèves à travers les écrits d'invention. 
Par l'écrit, on doit entendre au sens large «tout système sémiotique visuel et spatial ; au sens étroit, c'est un système graphique de notation du langage. » (Ducrot et Todorov, 1979, p.254). Par ailleurs, pour ce qui est du mot invention, il désigne étymologiquement l'action de trouver, de découvrir, il est « un emprunt ancien au latin classique inventio » (Rey, 2010, p.5029).

Le dictionnaire linguistique considère à lui aussi que l'invention est la «première partie de la rhétorique, l'invention traite de la recherche des moyens nécessaires pour convaincre et persuader: ce sont les preuves, qui démontrent la vérité des assertions, les mœurs (ithos), qui attirent la bienveillance de l'auditoire, les passions (pathos), par lesquelles on touche le cœur. » (Dubois et al., 2002, p.311).

L'écriture d'invention est une activité scripturale libre, on rejoint alors la conception proposée par Freinet, puisqu' « un texte libre doit être vraiment libre. C'est-àdire qu'on écrit quand on a quelque chose à dire, lorsqu'on éprouve le besoin d'exprimer, par la plume ou le dessin ce qui bouillonne en nous. " (Freinet, cité dans Bishop, 2005, p.145).

L'écriture d'invention tire un grand profit de la séquence narratologique, cependant certaines limites applicationnistes de cette pratique ne font que perdurer au sein de la classe marocaine, puisqu'un récit en classe de français est amené à longer et à suivre à la lettre, le schéma narratif suivant : situation initiale, élément perturbateur, péripéties et dénouement, et situation finale. Il faut entendre aussi et surtout par le mot applicationniste, cette " attitude qui consiste à importer dans les disciplines scolaires les savoirs savants ou académiques, le plus souvent récents, sans aménagement particulier ni réflexion spécifique.» (Le Goff, 2008, p.20).

Le récit perd subséquemment à cause de cette assiduité formelle et rigide, sur deux niveaux substantiels: un apport d'apprentissage humain conséquent, et une pratique constitutive et consécutive capitale.

L'enseignant dicte alors à travers une consigne volontariste et bien déterminée à l'avance, à faire écrire des textes qui donnent beaucoup d'importance à la structure linguistique (grammaire, orthographe...) par rapport aux autres aspects fondamentaux relatifs à l'écrit. On risque alors de ce fait de cantonner l'écriture dans une définition restreinte, à savoir une écriture entendue comme produit et non comme processus, une écriture qui capitalise des savoirs pré-construits au lieu d'être un espace d'expérimentation et de construction de savoirs et savoir-faire, une écriture où le sujet scripteur ignore le sujet lecteur singulier qu'il est également. (Oullet, 2011, p.17).

Par contraste à ce constat, l'imaginaire comme un vecteur psychoaffectif porte alors sur une indéniable interprétation de soi, de l'autre et même du monde, et qui fait largement parti de ce qui est occulté et mis à l'écart, au profit d'une forme textuelle qui n'est pas toujours indispensable au récit propre au cycle primaire.

Les travaux qui ont traité des écrits d'invention au niveau du collège et du lycée sont riches et nombreuses (Petitjean, 2003). Cependant peu d'études sérieuses sont consacrées au cycle primaire, et plus particulièrement pour ce qui est de la dernière année d'étude (6e année de ce même cycle), d'où l'importance, la teneur voire l'utilité de cette recherche.

\section{MÉTHODE}

Nous avons invité les élèves de la sixième année du cycle primaire d'une école à Rabat au Maroc, à rédiger et à écrire un récit d'imagination sur la base de la consigne suivante: «Raconter librement tout ce que vous voulez dans 30 lignes au grand maximum ».

On a tenu à expérimenter ce dispositif en deux classes distinctes, qui compte 20 apprenants pour chaque classe correspondante à la sixième année. Dans ce niveau terminal du cycle primaire et qui permet à l'apprenant une fois réussi de passer directement au cycle collégial. On peut alors élaborer et repartir sagement et après un grand processus d'observation éducative pour les deux classes, de 40 élèves, en trois catégories différentes : (1) des élèves en grande difficulté d'écriture, (2) des élèves ayant une maîtrise passable de la langue, 
mais démotivés par rapport à l'écriture en classe ou en dehors de la classe, et (3) des élèves bons en français et qui écrivent régulièrement en classe et en dehors de la classe.

Le traitement accordé aux écrits des élèves se fera prudemment en trois temps différents dans la forme, mais qui sont totalement complémentaires dans le fond et qui façonnent intrinsèquement le dispositif expérimenté: (a) une analyse des lacunes commises par les apprenants en termes de forme et de contenu, et qui sont propres à leurs uniques productions écrites, (b) l'entame d'une discussion et d'une conversation franches avec les élèves sur leurs difficultés et achoppements rencontrés au cours de leurs productions écrites initiales, et ce pour en résoudre et les dépasser. Mais il y a aussi une invitation adressée à l'élève en deuxième lieu, pour réécrire à nouveau le même texte pour des fins d'amélioration et de perfectionnement continus, et (c) une correction finale en groupe et avec une troisième tentative de réécriture.

La réécriture prend toute sa subjugation au sein de ce dispositif, puisque le Goff s'est efforcé par l'entremise de cette pratique, à interroger cinq dogmes largement partagés par les apprenants :

(1) un dogme conceptuel qui réduit l'écriture à un objet de médiation, au service de l'expression et de la représentation, (2) un dogme cognitivoscriptural qui entérine l'idée que la pensée précède l'écriture et que l'écriture n'est qu'une opération de mise en mots d'un déjà-là, préconçu intellectuellement, (3) un dogme esthétique qui reconnaît les qualités formelles d'une production écrite en fonction de critères normatifs qui fondent le bien-écrire, (4) un dogme artistique qui interprète la singularité, l'originalité d'un écrit comme étant l'expression du don et de l'inspiration, et non le résultat d'un travail et des influences intertextuelles, et enfin (5) un dogme téléologique qui valorise le produit achevé et moins le processus de génération de l'écriture. (2006, p.17).
Deux hypothèses centrales et sousjacentes orientent alors ce travail de recherche, puisqu'on bascule d'une hypothèse qui confirme l'importance de la consigne, comme source d'émancipation de l'imaginaire au sein des écrits des élèves et pour l'autre, qui donne peu d'importance à la consigne qui stipule et oriente vers de l'écriture libre, comme une mine profonde d'inspiration de l'imaginaire au sein des textes des élèves.

\section{RÉSULTATS ET DISCUSSION}

L'analyse des productions écrites [1] des élèves a permis de dégager et de mettre en avant trois groupes distincts: (a) G1 : le premier groupe mobilise les 12 textes d'apprenants, et qui ont commis des erreurs sur deux niveaux, que ce soit sur le plan formel, mais aussi sur le plan de l'imaginaire, (b) G2 : un deuxième groupe qui rassemble 8 textes, dont les apprenants ont excellé sur le plan formel, mais qui n'ont pas réussi malencontreusement en valeur créative, et (c) G3: le troisième groupe réunit 20 textes riches en imaginaires, mais avec un certain nombre de lacunes de surface assez déterminantes.

Le premier groupe G1, qui se compose de 12 textes, compte des apprenants qui ont des difficultés sur le niveau formel du récit, mais qui ont aussi une construction de l'imaginaire qui laisse grandement à désirer, puisqu'on trouve une absence totale de ce dernier point.

Une flagrante incohérence organisationnelle et une inconséquence très expressive se manifeste malgré l'âge avancé de l'enfant, et qui ne fait ici que raconter des faits et des anecdotes quotidiennes portant sur une réalité vécue sans la moindre production d'un récit d'imagination.

Les grandes étapes $\mathrm{du}$ récit sont mélangées de façons inquiétantes, voire inexistantes par moment, avec des protagonistes qui sont mal placées et incorrectement fonctionnalisées, et sur le volet microstructurel, les productions écrites se caractérisent pleinement par une intrigue qui est peu, voire nullement développée, avec une mauvaise ponctuation et un anachronisme inquiétant. 
Le deuxième groupe G2 rassemble 8 textes homothétiques, et qui se distinguent copieusement du premier groupe de textes réalisés par les apprenants, et c'est surtout sur le plan de la macrostructure du récit que l'abime apparait clairement avec une quasiabsence en contradiction et en non-sens. Cependant, on observe clairement la nonprésence de l'imaginaire dans les textes des élèves et on remarque en effet, une centration poussée davantage sur la forme que sur le contenu.

Ceci peut nous amener à penser qu'un bon nombre d'apprenants trouvent du mal à rédiger des textes qui peuvent maintenir un équilibre entre le pôle formel et qui est apparemment si cher à l'école marocaine, et le pôle imaginaire qui demeure une pratique très importante en didactique de l'écrit.

Le troisième groupe G3 réunit les 20 textes qui se distinguent amplement $\mathrm{du}$ premier et du deuxième groupe, et qui sont réunis par concordance en G1 et en G2.

Puisque les récits du troisième groupe sont marqués par l'existence et la présence, qui est forte importante du pôle imaginaire, et dont les élèves ont réussi habilement à joindre et à combiner avec leurs faits et actes journaliers. Cependant on peut relever la présence d'une accumulation et d'une succession d'idées sans le moindre agencement raisonnable, et qui peut s'expliquer par l'absence d'une véridique phase de réflexion et de planification qui précède la production et l'écriture des textes réalisés et entrepris par l'apprenant.

On peut noter aussi la présence quasi dominante de certains personnages imaginaires qui sont extraits des dessins animés et des films consacrés aux enfants et qui sont pleinement en vogue ces jours-ci, avec des évènements qui se rapprochent de l'histoire du film et de l'anime par moment.

Bien évidemment des erreurs de langues et de structures persistent, cependant l'apprenant se voit libérer des chaines évaluatives que comprend la consigne et trouve apparemment un plaisir fou à écrire, voire à dépasser et à surpasser largement les 30 lignes recommandées.

Pour ce qui est du deuxième volet $d u$ dispositif [2] et qui est relatif à ce stade au travail de réécriture, il vise premièrement à faire apprendre aux élèves que l'écriture est une activité qui ne s'improvise nullement, et qui exige par conséquent certaines pratiques nécessaires et obligatoires pour mener à bien leurs travaux rédactionnels.

La démarche d'écriture que l'apprenant devrait adopter et pratiquer par l'entremise de l'activité de réécriture se compose ainsi en 5 points essentiels : (1) la mise en œuvre des savoirs et des savoir-faire antérieurs, (2) la planification de l'écriture, (3) la structuration de la pensée, (4) la mise en texte, et (5) la révision.

Ce cinquième point qui est mis en avant (la révision), représente en effet de la réécriture proprement dite, de l'autocorrection, et de l'autoévaluation qui saurait devenir en un automatisme, une accoutumance scripturale spontanée, suite à cette approche didactique de réécriture assistée par l'enseignant.

La démarche de réécriture qui est considérée à ce niveau comme une pratique d'enseignement, a permis ainsi d'améliorer à $80 \%$ les textes proposés par les apprenants en 6e année du cycle primaire, et qui ont introduit le pôle d'imagination au sein de leurs propres récits, pour les textes qui ont été bien évidemment méticuleusement mis en examen.

Car si les fautes propres à la langue persistent et perdurent suite à l'activité de réécriture après explication détaillée de l'enseignant avec un pourcentage d'environ $60 \%$ pour les apprenants qui conservent ce type de fautes. Cependant ces lacunes tendent aussi et subséquemment à se réduire, pour presque $40 \%$ des apprenants sujets à examen, ce qui prouve indubitablement la validité didactique de cette démarche.

Prendre beaucoup de recul, d'écart et de distanciation face à son texte dans ce processus de réécriture permet à l'apprenant de remettre pleinement en cause ses avancées, ses connaissances et ses savoirfaire, mais aussi sa propre stratégie poursuivie. La phase de découverte reste alors le moment le plus important dans cette démarche, puisqu'il permet de prendre conscience des fautes commises, y remédier 
et surtout de développer une posture réflexive face à l'écriture.

L'utilisation du dictionnaire est d'une importance capitale pour un apprenant qui commence à douter grâce à cette démarche de l'orthographe utilisée, et qui s'efforce à le perfectionner au fur et à mesure de son autocorrection. Un dispositif d'enseignement brillant qui a su alors impliquer sérieusement l'élève dans son propre et unique processus d'apprentissage.

Pour ce qui est du troisième volet [3] propre au dispositif expérimenté, et qui est relatif à la correction en groupe des textes réalisés par les élèves, et dont on ne peut être que de plus en plus satisfait du résultat obtenu.

Car si auparavant avec la deuxième démarche de réécriture, on trouvait du mal à faire intégrer l'imaginaire au sein de tous les textes présentés par les élèves (seulement $80 \%$ ), ceci prend une autre tournure avec la correction en groupe. Puisqu'on constate, que presque la totalité des textes ont pu intégrer de l'imaginaire après une troisième réécriture et qui a précédé la correction collective des récits.

Il faut entendre par correction collective une correction purement formative, qui est administrée et gérée par les paires, et dont on a fait appel pour perfectionner davantage le dispositif qui est mis en examen.

Un scénario d'apprentissage qui fait que l'apprenant qui a su intégrer l'imaginaire prend en tutelle celui qui n'a pas réussi à le faire, en l'accompagnant dans cette troisième et dernière tentative pour rédiger en bonne et due forme, un texte qui intègre pleinement le plan imaginaire dans ses moindres recoins. L'évaluation s'est faite alors par d'autres apprenants pour rendre la démarche plus efficace et plus efficiente.

La grille de relecture et de correction nous a été $\mathrm{d}^{\prime}$ une grande utilité, puisqu'elle a permis d'unifier la correction entamée par l'apprenant et de la cibler vers un but assez précis, correspondant aux quelques éléments structurels qui sont très bien prescrits, et avec une effective implication de l'imaginaire.

Par ailleurs, on peut constater que si on a réussi à intégrer en troisièmes tentatives, l'imaginaire en totalité et dans la majorité des écrits, on n'a nullement pu et su améliorer pleinement et significativement l'aspect formel et structurel de la langue au niveau de la troisième étape de ce dispositif, comparaissant fait avec la deuxième phase, et avec ce qui s'est réalisé et élaboré de bénéfique dans ce sens.

\section{CONCLUSION}

La complexité de l'acte d'écriture en langue française, impose désormais à considérer amplement l'imaginaire au sein de la classe primaire, en advenant une nécessité absolue en didactique de l'écrit. Le dispositif expérimenté a permis ainsi de mettre en valeur ce plan au sein des récits produits par les élèves et de confirmer par conséquent la première hypothèse, c'est ainsi donc qu'une bonne reformulation de la consigne proposée aux apprenants est un facteur déterminant pour intégrer l'imaginaire dans leurs textes respectifs.

Notre contribution consiste aussi à expliquer les difficultés et les lacunes des élèves en cycle primaire selon leurs attachements au contenu ou à la forme de leurs propres textes, et de faire parler subséquemment les apprenants de leurs propres difficultés et achoppements afin qu'ils puissent prendre conscience de leurs lacunes respectives (un processus métacognitif), en essayant ensuite de les faire travailler en groupe dans le but de renforcer davantage l'efficacité et l'efficience de notre propre approche d'enseignementapprentissage.

En effet, l'intérêt de cette approche repose à repenser et à remettre en cause les pratiques d'évaluation courantes en classe de français et de permettre autrement aux élèves $\mathrm{du}$ cycle primaire, de prendre plaisir à l'écriture et de vivre partiellement l'expérience des « écrivains » en revalorisant sérieusement l'acte de réécriture comme processus dynamique. C'est ce processus de réécriture et qui fait partie intégrante de cette approche, qui constitue le gage permanent d'une prise en compte didactique de l'imaginaire en classe de français.

À l'autre versant, il nous paraît utile et opportun de mettre l'accent in fine, sur les 
trois enjeux relatifs à la mise en place de ce dispositif, et ce qui nous semble particulièrement salutaire à l'égard de l'intervention didactique en classe de Français.

Le premier point fondamental consiste à modifier les pratiques d'écriture et d'évaluation y afférentes, en ébauchant sur une véridique modification du fonctionnement classique des consignes d'écriture, et en mettant alors en place et de façon pertinente, des dispositifs d'aide et d'accompagnement des activités entreprises par des apprenants, et dont la réécriture et qui est totalement mis en exergue ici, peut être retenu comme un cas et un exemple parlant.

Le second élément est qui ne manque nullement d'intérêt, consiste par conséquent à expliciter les objectifs et les attentes selon le type de récit sollicité, et ce en revalorisant le rôle fonctionnel de l'imaginaire dans l'écriture.

Le troisième enjeu majeur consiste à son tour, à interroger davantage le statut du récit en classe de français, à revisiter les différents exercices qui s'y rapportent, et à rompre avec les formes figées et standardisées auxquelles on a tendance à se rattacher vainement.

\section{REMERCIEMENTS}

Nous remercions particulièrement toute personne ayant pris part à la lecture, à l'amélioration et à la publication éventuelle du présent article.

\section{RÉFÉRENCES}

Bishop, M.-F. (2005). Texte libre et écriture d'invention, quel rapport?. Pratiques, 128(1), 143-153.

Blaser, C. et Chartrand, S. (2008). Le rapport à l'écrit : un outil pour enseigner de l'école à l'université. Namur, Belgique: Presses universitaires de Namur.

Delcamber, I. (2007). Du sujet scripteur au sujet didactique. Le français aujourd'hui, 157(2), 33-41.

Dubois, J., Giacomo, M., Guespin, L., Marcellesi, C., Marcellesi, J.-B, Mevel, J.-P. (2002). Dictionnaire de linguistique. Paris, France : Larousse.

Ducrot, O. et Todorov, T. (1979). Dictionnaire encyclopédique des sciences de langage. Paris, France : Seuil.

Le Goff, F. (2008). Réflexions sur la réécriture en écriture $\mathrm{d}$ 'invention. Recherches et travaux, 73(1), 19-34.

Le Goff, F. (2006). Écriture d'invention, Réécriture et enseignement de la littérature (Thèse de doctorat inédite). Université Paul Verlaine.

Oullet, S. (2011). Le sujet lecteur et scripteur: Développement d'un dispositif didactique en classe de littérature (Thèse de doctorat inédite). Université de Toulouse.

Petitjean, A. (2003). Histoire de l'écriture d'invention au lycée. Pratiques, 118(1), 181-207.

Reuter, Y. (2000). Enseigner et apprendre à écrire. Paris, France : E.S.F.

Rey, A. (2010). Dictionnaire historique de la langue française. Paris, France: Le Robert. 